\title{
Perfiles empresariales y participación de los trabajadores con cualificaciones intermedias en actividades de innovación: el caso del País Vasco
}

\section{Small and Medium Profiles and Intermediate Worker Participation in Innovation: The Case of the Basque Country}

*Cristina Lavía

Departamento de Sociología y Trabajo Social. Universidad del País Vasco (UPV/EHU). España / Spain cristina.lavia@ehu.eus

\section{Beatriz Otero}

Departamento de Sociología y Trabajo Social. Universidad del País Vasco (UPV/EHU). España / Spain beatriz.otero@ehu.eus

Recibido / Received: 21/10/2015

Aceptado / Accepted: 16/04/2016

\section{RESUMEN}

El objeto de este trabajo es analizar la participación de los trabajadores con perfil de formación profesional (FP) en las actividades de innovación que se desarrollan en las pequeñas y medianas empresas vascas e identificar qué características de las empresas se asocian diferencialmente con tal participación. Para ello, se ha efectuado un análisis de las respuestas a una encuesta obtenidas de una muestra aleatoria de 330 empresas industriales del País Vasco. Los resultados desvelan importantes niveles de participación de los trabajadores FP en los procesos de innovación. La mayor intensidad de tal participación se tiende a producir entre las pymes metalmecánicas de menor nivel tecnológico y tamaño pequeño, que cuentan con plantillas de FP en puestos del organigrama dedicados al desarrollo técnico/ tecnológico, y presentan altos niveles de innovación y cooperación. Estos resultados sugieren que el sistema educativo de FP realiza una contribución importante a la competitividad de estas PYMEs.

Palabras clave: Formación profesional, innovación, capital humano, pyme, industrial.
Eneka Albizu

Departamento de Economia Financiera II. Universidad del País Vasco (UPV/EHU). España / Spain eneka.albizu@ehu.eus

Mikel Olazaran

Departamento de Sociología y Trabajo Social. Universidad del País Vasco (UPV/EHU). España / Spain mikel.olazaran@ehu.eus

\section{ABSTRACT}

The aim of this study is to analyze Vocational Education and Training (VET)-level employee participation in the innovation processes of industrial small and medium enterprises (SMEs), with a view to indentifying the characteristics of those firms which show the highest levels of such participation. The results are based on a survey to a representative sample of 330 SMEs from the Basque Country, a leading region in VET terms in Spain. Important levels of intermediate worker participation in innovation emerge, mainly in small, innovative firms from metal and low technology sectors. Other characteristics of high participation firms are an important presence of intermediate technicians and workers in technology development areas and high levels of external cooperation in innovation. The evidence gathered shows that the VET system, as provider of intermediatelevel skills, makes an important contribution to the competitiveness of Basque industrial SMES.

Keywords: Vocational education and training, innovation, human capital, industrial small and medium enterprises.

\footnotetext{
*Autor para correspondencia / Corresponding author: Cristina Lavía Martínez. Departamento de Sociología y Trabajo Social. Facultad de Ciencias Sociales y de la Comunicación. Universidad del País Vasco/Euskal Herriko Unibertsitatea (UPV/EHU). Barrio Sarriena s/n. 48940 Leioa (Bizkaia). Sugerencia de cita / Suggested citation: Lavía, C., Otero, B., Albizu, E., Olazaran, M. (2016). Perfiles empresariales y participación de los trabajadores con cualificaciones intermedias en actividades de innovación: el caso del País Vasco. Revista Española de Sociología, 25 (3): 367-386. (http://dx.doi.org/10.22325/fes/res.25.3.2016.367)
} 


\section{INTRODUCCIÓN}

La formación de los trabajadores ha preocupado históricamente a empresarios, sindicalistas y académicos, dado que el capital humano es una de las principales bases del desarrollo económico y social. Sin embargo, resulta paradójico constatar que la Formación Profesional (FP) ha representado en España una alternativa formativa a la que se ha prestado muy poca atención por parte de la Administración y ha caído, incluso, dentro de cierto descrédito social, estando asociada a profesiones y perfiles sociales considerados tradicionalmente bajos ${ }^{1}$.

En el análisis sobre la expansión de la educación y mercado de trabajo efectuado en cinco importantes países europeos (Alemania, Reino Unido, Francia, Italia y España), Béduwè y Planas (2003) aportan evidencias de las importantes diferencias existentes entre estos países desde mediados del siglo xx. Por ejemplo, Alemania casi triplica y Reino Unido supera en un $50 \%$ las tasas de población con estudios profesionales iniciales en la generación nacida en la década de los setenta, que en España es próxima al $20 \%$ de la población en edad de cursar estos estudios. Esta tasa no se ha modificado sustancialmente desde entonces, poniendo de manifiesto una carencia en la articulación entre el sistema educativo y productivo en España.

En todo caso, una economía que se desarroIla crecientemente a través de empleos basados en el conocimiento, con contenidos ocupacionales rápidamente cambiantes, cada vez más requerirá de personas trabajadoras con competencias que se adquieran a través de la formación continua y partiendo de la base de una formación incial só-

1 Distintos estudios ponen de manifiesto la relación existente entre la adquisición de cierto prestigio social y el cursar estudios superiores. Shavit and Muller sugieren (1998, pp. 19-20) que, en Europa, el acceso a posiciones sociales más destacadas está fuertemente influenciado por el nivel de cualificaciones adquirido. Así, España no ha sido ajena a un proceso - que también se ha dado en otros países europeos - de aumento de los niveles formativos de su población durante las últimas décadas. Sin embargo, los porcentajes de población con estudios de FP difieren considerablemente de país a país. lida, un prerrequisito esencial para la formación a lo largo de la vida (Planas et al., 2004). Así, las competencias de las personas con estudios de FP juegan, hoy más que nunca, un papel crucial en la competitividad empresarial. Tal y como ponen de manifiesto Toner et al. (2004: 27), las personas con estudios de FP contribuyen a la generación de mejoras de productividad, innovaciones de producto y proceso, así como a la creación y difusión de nuevo conocimiento. Las autoridades de la Administración han entendido este tema y en los últimos años están impulsando cambios que propician una más eficaz adaptación de las competencias de las personas trabajadoras a las necesidades de las empresas y a la búsqueda de empleo impulsando soluciones como: la formación dual, el impulso del emprendizaje, el desarrollo del Sistema Nacional de Cualificaciones para el reconocimiento de competencias, el e-learning, etc. En este contexto, la proposición general que se propone contrastar en este trabajo es que los trabajadores con cualificaciones intermedias (FP) juegan un papel importante en las actividades de innovación empresarial.

Siendo la relación existente entre el sistema de FP (reglada y continua) y los procesos de innovación empresarial un objeto de estudio relevante por las razones aducidas, se trata de una relación escasamente estudiada (Moodie, 2006). Este trabajo pretende efectuar una aportación en torno a este tópico. Más concretamente, el estudio pretende dar respuesta a las siguientes dos principales preguntas de investigación: ¿participan efectivamente los trabajadores con cualificaciones de FP en los procesos de innovación empresarial?; y, si es así, ¿qué características presentan las empresas en las que esto sucede? Para dar respuesta a estas cuestiones, nos serviremos de los resultados de una encuesta efectuada ad hoc entre pymes industriales del País Vasco.

El trabajo se estructura a través de los siguientes apartados. En primer lugar se efectúa una revisión teórica sobre el objeto de estudio para, a continuación, efectuar una breve presentación del caso que vamos a analizar (el País Vasco). Posteriormente, se explica la metodología de recogida y tratamiento de los datos. Se expondrán después los resultados obtenidos, para finalizar con el establecimiento de conclusiones. 


\section{MARCO TEÓRICO}

Desde la perspectiva del Sistema Regional de Innovación (SRI), la creación y aplicación de conocimiento en actividades económicas es un proceso localizado, condicionado por factores contextuales específicos. Así, se considera que elementos como, por ejemplo, los clusters industriales existentes, la cultura técnica común desarrollada a través de procesos de co-aprendizaje, las agencias de soporte a la innovación creadas, la oferta educativa y los niveles formativos de los trabajadores, las relaciones entre el sistema productivo y educativo, etc. condicionan y pueden favorecer las interacciones que propician el intercambio de conocimiento tácito y un ahorro en los costes de transacción entre los actores del sistema.

En última instancia, la perspectiva del SRI asume que el conocimiento reside en las personas y en las relaciones sociales que se producen entre éstas, y que es dependiente de un contexto y "adherido" a un territorio (Navarro, 2009). Por tanto, los factores que inciden en la conformación de un SRI son las precondiciones existentes para la innovación empresarial (Kaufmann y Tödtling., 2000). En el ámbito regional la mayoría de las empresas tiene un tamaño pequeño. Por tanto, tienen acceso a una base limitada de recursos (humanos, financieros, tecnológicos, gestión, etc.), lo cual condiciona sus posibilidades de realizar actividades de investigación y desarrollo (I+D) y de establecer relaciones de cooperación con otros agentes.

El concepto de "capacidad de absorción" (Cohen y Levinthal, 1990; Zahra y George, 2002) cobra importancia en este contexto. La "capacidad de absorción" es la habilidad de reconocer el valor de una información, asimilarla y destinarla a fines comerciales que desarrolla una empresa (Cohen y Levinthal, 1990: 128). Las fases de "adquisición" y "explotación" del conocimiento que posibilitan la innovación están condicionadas por las relaciones que mantiene la empresa con el exterior (fuentes externas de conocimiento y complementariedad del conocimiento propio con el de otros actores - proveedores, clientes, centros de educación e investigación, etc.-). Por otro lado, la "asimilación" y "transformación" del conocimiento son procesos internos que estarán condicionados por las relaciones que se establezcan entre diferentes unidades organizativas 0 equipos humanos que, a través de diferentes mecanismos de integración social, comparten conocimiento y permiten la búsqueda de nuevas soluciones a los problemas que tiene planteados la empresa para adaptarse a los requerimientos de sus clientes. El planteamiento teórico de la "capacidad de absorción" se imbrica con otros enfoques más recientes como el de la "innovación abierta" (Chesbrough, 2003) que sugiere que las empresas necesitan abrir sus procesos de intercambio de conocimiento con el exterior, combinando las tecnologías y conocimientos generados tanto en el interior de la empresa como fuera de la misma, para acelerar los procesos de innovación y comercialización de las mismas.

Entre los factores contextuales espaciales que inciden en los procesos de innovación de las pymes cobra especial importancia la formación de los trabajadores (Lundvall y Lorenz, 2010). En un interesante estudio, Lorenz y Valeyre (2006) comparan la forma de trabajar (sistemas organizativos) y de aprender que se presentan mayoritariamente en diferentes países europeos apreciándose diferencias entre países, especialmente entre los países nórdi$\cos y$ (en menor medida) centroeuropeos y los del sur. En un estudio posterior, Lundvall, Rasmussen y Lorenz (2008) relacionan la formación "discrecional" 0 continua ofrecida por la empresa a sus trabajadores — parte de la cual estará destinada a renovar las capacidades y conocimientos técnicos precisados para desarrollar innovación de productos y procesoscon el nivel formativo de los trabajadores (estudios superiores y formación profesional, respectivamente) en los mismos países europeos. Además de volver a reflejar situaciones diferenciadas entre países (y sistemas educativos), uno de los principales hallazgos de este estudio estriba en que la formación continua en el trabajo está notablemente correlacionada con la formación profesional y bajamente relacionada con los estudios superiores. Por tanto, la formación continua discrecional aporta recursos complementarios claves para la exploración del conocimiento y la innovación cuando se imparte a personas que han efectuado estudios de formación profesional.

La mayoría de las innovaciones que efectúan las pymes son incrementales y están basadas en el modo de innovación DUI (doing, using, interacting). Es decir, a medida que los trabajadores se enfrentan a nuevos problemas/proyectos que plantean los 
clientes tienen que encontrar soluciones que van haciendo desarrollar su base de know how. Por tanto, para facilitar este proceso de aprendizaje individual y colectivo, la empresa debe poner en marcha sistema de organización que favorezcan el aporte y transferencia de conocimiento de los trabajadores.

Lundvall y Lorenz (2010: 61-62) sostienen la tesis de que la interacción con los clientes y las prácticas organizativas (como equipos de proyecto, los grupos de resolución de problemas, la rotación de trabajos y tareas), promocionan el aprendizaje y el intercambio conocimiento, contribuyen al desempeño de la innovación. Los equipos de trabajo constituyen el ámbito ideal para generar, compartir y acumular conocimientos (Nonaka, 1994; Janz y Prasarnphanich, 2003). Por tratarse éstos - generar, compartir, acumular conocimiento - de procesos sociales, el ámbito del equipo de trabajo posibilita la generación de la confianza, los lazos personales y el compromiso colectivo que facilita su desarrollo. La autonomía e interdisciplinariedad de los equipos de trabajo favorece la transferencia de conocimiento (García-Pinto, A. et al., 2010).

Los equipos de trabajo estables son responsables de procesos de adición de valor y definen sus propias metas, los mecanismos de organización (dentro del contexto de la empresa) y los de control. Como señalan Bolland y Hofer (2001: 230), "en las empresas de alta tecnología la centralización se ha desplazado de las máquinas a los grupos de trabajo, lo que representa un viraje del culto de la producción a la dinámica de personas que trabajan con otras personas y a la prestación de servicios". Pérez y Quevedo (2006) sugieren que la mayoría de las compañías innovadoras españolas organizan sus actividades de $1+D$ en equipos de trabajo mientras que Jiménez-Jiménez y Sanz-Valle (2008) confirman la relación existente entre la participación de los trabajadores y el trabajo en equipo con el desarrollo de actividad innovadora.

\section{MARCO REGIONAL: PAÍS VASCO}

De acuerdo con el objeto de este estudio, la Comunidad Autónoma del País Vasco (CAPV) es una región que podemos caracterizar diferencialmente por dos elementos: cultura y desarrollo industrial, y alto nivel de desarrollo de los estudios profesionales.

\section{Cultura y desarrollo industrial}

En relación con la primera cuestión, cabe señalar que la tradición industrial-metalúrgica del País Vasco se hunde en el tiempo y, en la actualidad (Eustat, 2015), ésta continúa siendo una región marcadamente industrial: el $21,7 \%$ de su PIB se obtiene de actividades industriales, mientras que en el nivel estatal y europeo (EU-28) el PIB industrial es del 16,1 y $17,2 \%$, respectivamente. El empleo industrial representa en la CAPV el 20,8\% del total, mientras que a nivel estatal y europeo (EU-28) supone el 12,5 y $15,6 \%$, respectivamente.

De acuerdo con su legado industrial, las manufactureras vascas fundamentalmente se concentran en actividades metalmecánicas (CNAE 09): Metalurgia, con 33,7\% del empleo industrial y $27,3 \%$ del Valor Añadido Industrial (VAI); Maquinaria y Equipo, con el 10,4\% del empleo industrial y 10,7\% del VAl; y Material de Transporte, que aporta el 9,1\% del empleo industrial y 10,4\% del VAI (Eustat, 2015). Estas tres ramas de actividad representan, aproximadamente, la mitad del aporte de la industria vasca, mientras que el resto de las actividades están bastante diversificadas.

En relación con el contenido tecnológico del sector industrial (\% VAB industrial), y utilizando la misma fuente de información (Eustat, 2015), podemos afirmar que resulta apreciable: el $4,4 \%$ de las actividades corresponde a nivel tecnológico alto; el 26,2\% a medio-alto; el 40,1\%, a actividades con nivel medio-bajo; y el 14,3\% restante a actividades con bajo nivel tecnológico.

El gasto realizado por el conjunto de la industria en actividades de innovación tecnológica representa el 40,1\% del gasto total de la CAPV (Eustat, 2015). Por ramas, las más implicadas en actividades de innovación son — de más a menos—: Material de transporte, con un 33,9\% del total del gasto industrial en innovación tecnológica (fundamentalmente, invirtiendo en maquinaria); la Metalurgia y productos metálicos, con un $19,7 \%$ sobre el total del gasto industrial (destacando en I+D externa); Material y equipo eléctrico, con un $15,3 \%$; y, Maquinaria y equipo, con un $10,1 \%$ (focalizado muy notablemente a I+D interna).

\section{Desarrollo de la FP}

La FP cuenta con una larga tradición en el País Vasco como producto de una activa relación estable- 
cida entre el sistema productivo y el educativo. Así, las grandes firmas industriales se preocupan desde los inicios del siglo xx de dar formación a sus trabajadores a través de las escuelas de aprendices. Más tarde, en la época de desarrollismo industrial en España (segunda mitad del siglo xx), se produce una especialización educativa profesional en función de la distribución comarcal de la industria². El mismo movimiento cooperativo de Mondragón surge de la Escuela Profesional de la localidad que le da nombre en 1957. Finalmente, es el Gobierno Vasco quien asume competencias en Educación y regula e impulsa estos estudios atendiendo a las demandas de las empresas desde comienzos de los años 80. Como resultado de ello, se ha producido un importante crecimiento del porcentaje de personas ocupadas en la industria con cualificaciones de FP, pasando en el caso de la CAPV, del $12,1 \%$ en 1985 a cerca del $40 \%$ en 2010 (el doble aproximadamente de la media estatal, según datos facilitados por el Ministerio de Educación, Cultura y Deporte en 2014).

La FP en la CAPV se diferencia a nivel estatal por las siguientes características (Albizu et al., 2013):

a) Apoyo de la Administración ${ }^{3}$ y elevados niveles relativos de institucionalización (el Gobierno Vasco es el único gobierno regional que cuenta con una viceconsejería específica de FP);

b) Penetración de la FP Grado Superior (FPGS). Las tasas brutas de escolarización (MECD, 2014) en FP de Grado Medio (35,6\%) son similares a la media española $(35,4 \%)$, mientras que en FPGS $(49,7 \%)$ son notablemente superiores a la media estatal $(30,5 \%)$;

c) Matriculación de las familias industriales (aprox. 50\% de la matrícula) y, muy especialmente, en Fabricación Mecánica, Electricidad y Electrónica, y Mantenimiento y Servicios a la Producción;

d) Penetración de la enseñanza privada concertada (alcanza más del $50 \%$ de los centros y

2 P.e.: Cuenca media del Deba (armería), Tolosa (papel), Cuenca alta Urola (madera), Cuenca Deba (máquina-herramienta), Bergara (Textil/Estampación frío), Duranguesado (fundición), Cuenca del Bidasoa (química), Goi Herri (elevación), Cuencas Lea-Artibai-Oka (polímeros), etc.

3 Se puede encontrar una revisión general de los tres primeros planes de FP del Gobierno Vasco, que abarcan en su conjunto el período 1997-2013, en Albizu et al. (2013). estudiantes matriculados (un 20\% por encima de la media estatal) (Albizu et al., 2013: 200). Esta estructura de titularidad (balanceada al sector privado) puede ser uno de los elementos que facilita unas relaciones más estrechas y flexibles entre este sector educativo y el mundo industrial;

e) Presencia de centros integrales — con capacidad para desarrollar formación para el Empleo/ocupacional- (40\%) y fuerte participación de los mismos en la formación continua, suponiendo cerca del $50 \%$ de los recursos económicos gestionados a nivel autonómico en este concepto (Albizu et al., 2013: 206-207).

En la actualidad se encuentra en vigor el IV Plan Vasco de Formación Profesional (2014) que destina a la FP 89.280.771€ en 2015 y prevé destinar 101.192.123€ durante el ejercicio de 2016, donde se plantean las siguientes líneas de actuación: Formación Integrada (Funcionamiento, Equipamiento, Formación Inicial, Formación para el Empleo); Innovación Aplicada (Innovación en cooperación con las empresas, Metodologías activas de aprendizaje, Mejora continua); Emprendimiento Activo (Cultura emprendedora, Creación de empresas en centros de FP); Internacionalización de la FP vasca (Proyectos europeos, redes internacionales); e Infraestructuras. Con este plan el Gobierno Vasco pretende poner el foco en las PYMES, en la innovación, fomento de la cultura emprendedora y en el impulso para la creación de empresas en los centros de formación profesional.

Cabe señalar finalmente que la FP ha jugado un papel muy importante en el desarrollo económico y social de la CAPV: ha formado a cerca de un tercio de la población, dando importantes pasos para adecuarse a las necesidades de las empresas en el ámbito regional; ha mejorado la dotación de equipos humanos, infraestructuras y sistemas de gestión de los centros; ha impulsado y perfeccionado herramientas conducentes a la facilitación de la actividad innovadora en los propios centros de FP, así como en las empresas con las que los centros mantienen relaciones de cooperación, si bien continúa desligada de las infraestructuras de I+D (especialmente los centros tecnológicos y universitarios) potenciadas por el gobierno regional, con las cuales podría, a nuestro juicio, generar sinergias en su acercamiento a la facilitación de la innovación en las pymes vascas. 


\section{METODOLOGÍA}

\section{Recogida y tratamiento de datos}

Para dar respuesta a las cuestiones de investigación planteadas, se ha efectuado una encuesta telefónica ${ }^{4}$ a 330 pymes industriales (CNAE2009 05-39) del País Vasco de entre 10 y 250 trabajadores. Los datos se recogieron entre diciembre de 2013 y enero de 2014. La muestra se extrajo de una población real de 1.948 empresas (base de datos $\mathrm{SABI}$ ). Las personas encuestadas fueron Gerentes 0 Responsables de Recursos Humanos y/o formación y la encuesta se diseñó específicamente para estos estudios por el equipo investigador. Se trata de una muestra aleatoria estratificada proporcional por tamaños ( 5 estratos) que, para un Nivel de Confianza de $95 \%$ y bajo el supuesto más desfavorable $(p=q)$ asume un Error Muestral máximo del 4,9\%.

Los datos han sido tratados con SPSS v.22. Se han efectuado diferentes exploraciones multivariables entre las cuales se realizaron diversas regresiones stepwise para buscar la mejor combinación de variables explicativas sobre las variables originales que medían la participación de los trabajadores con perfil FP en las cinco formas de innovación investigadas. También se aplicó el análisis de correspondencias simples para representar algunas combinaciones de categorías de participación junto con otras características objetivas del funcionamiento de las pymes. Finalmente, la mejor representación de las relaciones detectadas se realizó mediante una regresión logística binaria efectuada sobre las características empresariales que suscitan diferencias entre pymes en cuanto a la participación de los trabajadores con perfiles de FP en actividades de innovación.

\section{RESULTADOS}

\section{Características de las empresas que conforman muestra}

4 Los contenidos del cuestionario se muestran como Anexo 1 por motivos de espacio ya que la encuesta tenía previstas numerosas aclaraciones de términos y precisiones sobre las que se dirigió específicamente el entrenamiento del personal de campo.
En primer lugar vamos a describir algunas características básicas de las empresas encuestadas y de la presencia de trabajadores con formación FP en ellas. Estas características se han tomado como variables independientes a la hora de explorar entre los posibles perfiles explicativos de la participación.

Como vemos en la tabla 1 , el perfil básico de las empresas encuestadas corresponde a pymes pequeñas (casi la mitad con menos de 25 trabajadores) y una distribución sectorial caracterizada por el peso de las actividades de Metalurgia y Fabricación de productos metálicos (CNAE09 códigos 24-25), que supone el sector de actividad del $40 \%$ de las pymes analizadas. Secundariamente, la fabricación de maquinarias $(21 \%)$ también representa una parte importante de la actividad industrial de las pymes vascas. Sumando ambas obtenemos el perfil mayoritario (60\%) de los sectores de metalmecánica. Además, de acuerdo con la clasificación Eustat de sectores en función de su intensidad tecnológica ${ }^{5}$, casi la mitad de las pymes encuestadas pertenecen a sectores de nivel medio-bajo y la mayoría (66\%) a los sectores medio-bajo y bajo. Un $31 \%$ de las pymes trabajan en sectores de intensidad tecnológica media-alta y alta. En principio, no hay interacción entre estas tres variables: tamaño, sector (metalmecánico frente a resto) y nivel tecnológico.

5 Empleamos la definición de Eustat de intensidad tecnológica para empresas industriales, que incluye cuatro niveles de sectores de actividad. Intensidad alta: Fabricación de productos farmacéuticos, Fabricación de productos informáticos, electrónicos y ópticos, Construcción aeronáutica y espacial. Intensidad media-alta: Industria química, Fabricación de armas y municiones, Fabricación de material y equipo eléctrico, Fabricación de maquinaria y equipo, Fabricación de vehículos, Fabricación de otro material de transporte, excepto Construcción Naval y Construcción Aeronáutica, Fabricación de instrumentos y suministros médicos y odontológicos. Intensidad media-baja: Reproducción de soportes grabados, Coquerías y refino de petróleo, Fabricación de productos de caucho y plásticos, Fabricación de otros productos minerales no metálicos, Metalurgia, Fabricación de productos metálicos excepto maquinaria y armas y municiones, Construcción naval, Reparación e instalación de maquinaria y equipo. Intensidad baja: Industria de la alimentación, bebidas, tabaco, textil y confección, Industria del cuero y calzado, Industria de la madera y del corcho y del papel, Artes gráficas, Fabricación de muebles, Otras industrias manufactureras. 
Tabla 1. Características de las empresas que conforman muestra

\begin{tabular}{|c|c|c|}
\hline Tamaño & $\mathrm{n}$ & $\%$ \\
\hline Hasta 24 empleados & 157 & 47,6 \\
\hline De 25 a 49 & 102 & 30,9 \\
\hline De 50 y más & 71 & 21,5 \\
\hline \multicolumn{3}{|l|}{ CNAEO9 } \\
\hline Metalurgia y Fabricación de productos metálicos (24-25) & 131 & 39,7 \\
\hline $\begin{array}{l}\text { Fabricación maquinaria y Equipos, Vehículos a Motor y Remolques, Otro material de } \\
\text { Transporte, Reparación de Maquinaria y equipo ( } 28-30 \text { y } 33 \text { ) }\end{array}$ & 70 & 21,2 \\
\hline Subtotal METALMECÁNICA & 201 & 60,9 \\
\hline $\begin{array}{l}\text { Industria del Papel, Artes Gráficas, Refino, Industria Química, Farmacéutica, Caucho, } \\
\text { Plástico, Productos minerales no Metálicos, Mueble, Otras industrias manufactureras } \\
\text { (17-23 y 31-32) }\end{array}$ & 50 & 15,2 \\
\hline $\begin{array}{l}\text { Industria extractiva, Industria de Alimentación, Bebidas, Tabaco, Textil, Cuero y } \\
\text { calzado, Madera, Corcho (05-16) }\end{array}$ & 47 & 14,2 \\
\hline $\begin{array}{l}\text { Fabricación de productos informáticos, electrónicos, ópticos, Material y equipos } \\
\text { eléctricos (26-27) }\end{array}$ & 28 & 8,5 \\
\hline $\begin{array}{l}\text { Producción y suministros de Energía (eléctrica, gas, vapor, aire acondicionado), } \\
\text { Suministro de agua, Saneamiento, Gestión de residuos, descontaminación (35-39) }\end{array}$ & 4 & 1,2 \\
\hline Subtotal RESTO sectores & 129 & 39,1 \\
\hline Nivel tecnológico & n & $\%$ \\
\hline Alto & 14 & 4,2 \\
\hline Medio alto & 89 & 27,0 \\
\hline Medio bajo & 154 & 46,7 \\
\hline Bajo & 65 & 19,7 \\
\hline NC & 8 & 2,4 \\
\hline \multicolumn{3}{|l|}{ Innovaciones que realizan } \\
\hline Innovación proceso & 213 & 64,5 \\
\hline Innovación producto & 238 & 72,1 \\
\hline Mejora procesos & 260 & 78,8 \\
\hline Mejora productos & 283 & 85,8 \\
\hline Nuevos sistemas organización & 267 & 80,9 \\
\hline Nuevos mercados & 276 & 83,6 \\
\hline Total & 330 & 100,0 \\
\hline
\end{tabular}

Fuente: Elaboración propia 
Con respecto a la presencia de trabajadores con formación FP en las plantillas, hay que decir que si bien todas las pymes analizadas cuentan con este tipo de personal, hay una gran variedad de tipos de plantillas en la muestra. Lo más común (58\%) es que los trabajadores FP constituyan menos de la mitad de la plantilla de estas pymes, lo cual implica que el otro $42 \%$ de casos puede calificarse de pymes con una presencia intensiva de trabajadores con esta formación. También se consignó en la encuesta la incidencia específica de la FP superior entre el conjunto de los trabajadores FP y resultó ser ya menor, aunque relevante: el $41 \%$ de las pymes no tiene ninguno o menos de una cuarta parte de FP superior entre sus trabajadores con FP, aunque otro $42 \%$ tiene al menos a la mitad con nivel superior.

Otra característica relevante es que se ha obtenido una muestra de pymes claramente innovadoras, tanto en alcance como en intensidad de formas de innovación. Como vemos, la realización de actividades de innovación estricta (producto, proceso, organización y mercados) o también de "mejoras" está realmente muy extendida entre las pymes vascas. Desde la innovación de producto, que se presenta como la forma más específica (64\% de las pymes), hasta la mejora de procesos ( $86 \%$ de las pymes), que aparece como la forma de innovar más "común", las tasas de realización de las actividades de innovación contempladas son realmente altas. Del total de empresas consultadas, además, solo un $10 \%$ no innova en absoluto, pero más de la mi$\operatorname{tad}(57,6 \%)$ han declarado que innovan en todas las formas consideradas.

Contamos también con otro indicador (subjetivo) de resultado en innovación para las pymes analizadas: preguntadas por su capacidad comparativa de innovación frente a competidores directos, un 30\% considera que está en "mejor" posición relativa como empresa innovadora que sus competidores; el resto "peor" (10\%) 0 "igual" (60\%). Más o menos ese $30 \%$ coincide con las pymes que realizan innovación de producto (la menos habitual entre las empresas de la muestra). A partir de ahí diferenciamos la mayor capacidad innovadora frente a la menor (los que se ven igual o peor) y esta percepción relativa (más innovadora 0 no) se relaciona con los demás indicadores de innovación componiendo la imagen que indicábamos al inicio de una muestra con perfil claramente innovador en extensión e intensidad: las empresas que tienen una mejor percepción de su capacidad innovadora frente a los competidores son las que realizan más tipos de innovación y viceversa.

\section{Grados de participación de los trabajadores FP en actividades de innovación (PTFPI)}

Para estudiar la participación de los trabajadores industriales y, concretamente, de los trabajadores con formación FP en actividades 0 estrategias de innovación, en la encuesta se han tenido en cuenta diversos aspectos. El aspecto central se ha considerado a partir de la medida más "directa" de participación en los distintos tipos de innovaciones según la clasificación presentada: producto, proceso, mejoras, organización y nuevos mercados ${ }^{6}$. Se ha preguntado hasta qué punto (nada, poco, bastante, mucho) los trabajadores FP participan en las distintas formas de innovación (cuando se dan). En prácticamente todas las formas de innovación (excepto en apertura de mercados) se produce, de modo mayoritario, "alguna" participación de trabajadores FP.

Ampliando los detalles y fijándonos en los niveles que reflejan una PTFPI más importante (bastante 0 mucha), descubrimos que cada tipo de innovación muestra una pauta diferente entre las pymes industriales vascas. La mayor participación del personal FP se produce en actividades de mejora (puede alcanzar al $51 \%$ de las pymes en mejoras de proceso y el $44 \%$ en mejora de producto). En actividades de innovación más estrictas,

6 La pregunta concreta fue: "Señale si en la empresa se realizan (referencia 4 últimos años) las actividades que se especifican y hasta qué punto los empleados con formación FP participan (nada, poco, bastante, mucho) en cada una de estas actividades: Innovación de productos, innovación de procesos, mejora de productos, mejora de procesos, nuevos sistemas de organización del trabajo, apertura de nuevos mercados". 
es algo menor y dispar: representa un $41,6 \%$ en innovaciones de proceso; un 33\% en innovaciones en organización y un $31,5 \%$ en innovaciones de producto. Si se trata de innovaciones comerciales llega únicamente al 18\% de los casos.

Al objeto de relacionar las formas de innovación y los grados de PTFPI se ha elaborado una clasificación de niveles 0 grados de participación de los trabajadores FP en las innovaciones. Este constructo resume las diversas combinaciones de situaciones en cuanto a formas de innovación y participación de los trabajadores de FP en ellas y diferencia también cuatro grados o niveles que se denominan igual que las categorías de las variables originales: nada, poca, bastante y mucha participación. La elaboración considera:

- Empresas con alta (mucha) PTFPI: empresas en las que los trabajadores de FP participan bastante 0 mucho en actividades de innovación de producto o proceso (actividades más estrechamente vinculadas a una definición estricta de innovación).

- Empresas con media (bastante) PTFPI: empresas en las que los trabajadores de FP participan poco o nada en innovación de producto o proceso pero, en cambio, participan bastante 0 mucho en el resto de actividades que desarrollan: mejora de productos, mejora de procesos, nuevos sistemas de organización o innovaciones de comercialización.

- Empresas con baja (poca) PTFPI: empresas en las que los trabajadores de FP participan algo en mejora de productos y/o mejora de procesos $y / 0$ nuevos sistemas de organización y/o innovaciones de comercialización.

- Empresas con ninguna (nada) PTFPI: empresas en las que las actividades de innovación se realizan sin participación ninguna de los trabajadores de FP.

La clasificación pretende discriminar en el continuum de situaciones que va desde las pymes en que no hay ninguna participación de $\mathrm{FP}$, aunque se desarrollen actividades de innovación (nada), hasta aquellas en que se produce una participación importante en las innovaciones de más peso y frecuencia (producto/proceso). Hay que tener en cuenta que las innovaciones 0 mejoras de producto $y / 0$ proceso presentan unas situaciones de participación bastante concordantes entre sí (rho Spearman entre 0,7 y 0,9).

Como vemos con esta nueva variable, la participación de los trabajadores de FP en los procesos de innovación es importante: casi un tercio de las pymes innovadoras desarrollan sus actividades de innovación con alta participación de sus plantillas de FP y no llegan a un $15 \%$ las pymes que las llevan a cabo sin ninguna intervención de estos perfiles profesionales. Es decir, respondiendo a la primera de las cuestiones planteadas en este trabajo, podemos decir que en las pymes vascas se desarrolla un nivel alto de innovación (tabla 1) y con una importante participación de los trabajadores FP en la misma (tabla 2).

Tabla 2. Grado de PTFPI

\begin{tabular}{l|c|c}
\hline & $\mathbf{n}$ & $\%$ \\
\hline Nada & 47 & 14,2 \\
\hline Poca & 79 & 23,9 \\
\hline Bastante & 69 & 20,9 \\
\hline Mucha & 102 & 30,9 \\
\hline Total (válido) & 297 & 100 \\
\hline
\end{tabular}




\section{Características empresariales y PTFPI}

Buscando perfiles característicos de empresas que se diferencien significativamente en cuanto a grado de PTFPI descubrimos que, en general, los grados de participación no se asocian directamente con las características descriptivas básicas de las empresas y de su actividad. Así las cosas, tomados de modo independiente, ni el tamaño, ni el sector, ni el nivel tecnológico de la actividad generan perfiles diferenciales de PTFPI.

Sin embargo, controlando efectos, descubrimos que hay algunas combinaciones más concretas de características descriptivas que muestran casos de mayor PTFPI. Es el caso de las pymes de sectores metalmecánica y de intensidad tecnológica baja 0 media baja (tabla 3): entre estas pymes es donde se puede apreciar que la mayor participación se produce en función del tamaño, concretamente, en las empresas más pequeñas. Este subgrupo de empresas que es el más característico de la industria vasca, pequeñas que trabajan en sectores de metalmecánica pero no tecnológicamente punteras, es precisamente donde se descubre diferencialmente la conexión entre innovación y FP: en el $41 \%$ de estas pymes la participación está en el grado más alto y solo un 10\% de estas empresas desarrolla innovaciones sin la participación de estos trabajadores.

\section{Porcentaje de trabajadores FP, ocupaciones y grado de PTFPI}

Aunque, como acabamos de señalar en el epígrafe anterior, la PTFPI no parece depender directamente del tamaño de las pymes industriales en el País Vasco, sí se relaciona con ciertos aspectos de composición de las plantillas. Más concretamente con la presencia y distribución de los trabajadores FP. Los resultados obtenidos sugieren que la PTFPI no depende del volumen global de plantilla FP, si bien, aparece significativamente asociada a una mayor presencia de personal con FP que han cursado grados superiores, así como a su trabajo en ocupaciones del tipo "técnico".

Tabla 3. Grado de PTFPI según tamaño controlando nivel tecnológico y sector

\begin{tabular}{|c|c|c|c|c|c|c|}
\hline \multirow[b]{5}{*}{ PTFPI } & \multicolumn{4}{|c|}{ Metalmecánica } & & \\
\hline & \multicolumn{4}{|c|}{ Nivel Tecnológico bajo o medio-bajo } & & \\
\hline & \multicolumn{4}{|c|}{ Tamaño* } & \multirow{2}{*}{\multicolumn{2}{|c|}{ Total }} \\
\hline & \multicolumn{2}{|c|}{ Menos de 50} & \multicolumn{2}{|c|}{50 o más } & & \\
\hline & $\mathrm{n}$ & $\%$ & $n$ & $\%$ & $n$ & $\%$ \\
\hline Nada & 10 & 10,0 & 7 & 28,0 & 17 & 13,6 \\
\hline Poca & 24 & 24,0 & 8 & 32,0 & 32 & 25,6 \\
\hline Bastante & 25 & 25,0 & 5 & 20,0 & 30 & 24,0 \\
\hline Mucha & 41 & 41,0 & 5 & 20,0 & 46 & 36,8 \\
\hline Total & 100 & 100,0 & 25 & 100,0 & 125 & 100,0 \\
\hline
\end{tabular}

${ }^{*} \mathrm{Chi}^{2}$ sig. $<0,05$

Fuente: Elaboración propia 
Tabla 4. Grado de PTFPI según volumen de plantilla FP, tasa de FP nivel superior y presencia de FP en departamentos técnicos

\begin{tabular}{|c|c|c|c|c|c|c|c|c|c|c|c|c|c|c|}
\hline \multirow{3}{*}{ PTFPI } & \multicolumn{4}{|c|}{ \% Empleados FP } & \multicolumn{4}{|c|}{ \% FP Superior } & \multicolumn{4}{|c|}{ FP en departamentos técnicos } & \multirow{2}{*}{\multicolumn{2}{|c|}{ Total }} \\
\hline & \multicolumn{2}{|c|}{$\begin{array}{c}\text { Menos de } \\
50 \%\end{array}$} & \multicolumn{2}{|c|}{$\begin{array}{c}\text { De } 50 \% \\
\text { más }\end{array}$} & \multicolumn{2}{|c|}{$\begin{array}{c}\text { Menos de } \\
50 \%\end{array}$} & \multicolumn{2}{|c|}{$\begin{array}{c}\text { De } 50 \% \\
\text { o más }\end{array}$} & \multicolumn{2}{|c|}{ SI } & \multicolumn{2}{|c|}{ NO } & & \\
\hline & $n$ & $\%$ & $n$ & $\%$ & $n$ & $\%$ & $n$ & $\%$ & $n$ & $\%$ & $n$ & $\%$ & $n$ & $\%$ \\
\hline Nada & 30 & 17,6 & 17 & 13,4 & 34 & 20,2 & 13 & 10,1 & 26 & 13,1 & 21 & 21,4 & 47 & 15,8 \\
\hline Poca & 47 & 27,6 & 32 & 25,2 & 49 & 29,2 & 30 & 23,3 & 47 & 23,6 & 32 & 32,7 & 79 & 26,6 \\
\hline Bastante & 39 & 22,9 & 30 & 23,6 & 36 & 21,4 & 33 & 25,6 & 45 & 22,6 & 24 & 24,5 & 69 & 23,2 \\
\hline Mucha & 54 & 31,8 & 48 & 37,8 & 49 & 29,2 & 53 & 41,1 & 81 & 40,7 & 21 & 21,4 & 102 & 34,3 \\
\hline Total & 170 & 100,0 & 127 & 100,0 & 168 & 100,0 & 129 & 100,0 & 199 & 100,0 & 98 & 100,0 & 297 & 100,0 \\
\hline
\end{tabular}

${ }^{*}$ Chi $^{2}$ sig. $<0,05$

Fuente: Elaboración propia

Los análisis efectuados no permiten detectar diferencias significativas de PTFPI entre las pymes cuando se considera el porcentaje de empleados con estudios de FP en la plantilla (tabla 4). Además, la distribución de la PTFPI es bastante similar en los dos grandes grupos de pymes atendiendo a la penetración de perfiles de FP (menos de 50\% de la plantiIla con FP y más de $50 \%$ de la plantilla con FP).

Sin embargo, una mayor o menor penetración de la FP superior en esta parte de la plantilla (con estudios de FP) sí se asocia con perfiles significativos de PTFPI. Así, entre las pymes con mayores porcentajes de FP superior en sus plantillas FP, la PTFPI es significativamente mayor que en las que tienen menores porcentajes de FP superior. En este colectivo (empresas con menos del $50 \%$ de los trabajadores FP con FP superior), existe el doble de empresas para las que la PTFPI es inexistente, que en aquellas en las que hay mayor proporción de empleados FP (10,1 vs. 20,2\%, respectivamente). En el otro extremo (mucha PTFPI), observamos que entre las pymes con más plantilla FP superior se llega a un $41 \%$ de mucha participación de los tra- bajadores FP, sensiblemente por encima del 29,2\% de PTFPI que se produce en las pymes con menores plantillas de FP superior.

Hemos analizado también la relación existente entre la trayectoria profesional, medida como nivel de especialización técnica/tecnológica logrado por los trabajadores de FP, y el grado de PTFPI. Así, se ha considerado la presencia de trabajadores con perfil FP en las actividades desarrolladas en departamentos técnicos tales como Departamento $I+D$, Oficina Técnica, y/o Departamento de Ingeniería de Fabricación y de Procesos. Consignando simplemente la existencia (Sí/No) de algún trabajador en los mencionados departamentos, observamos que la mayoría de las pymes cuenta con esa presencia (67\%) y el otro tercio no tiene ese tipo de trabajador 0 bien no tiene ninguna de esas estructuras. Como se deduce de los datos (tabla 4) esta variable es altamente discriminatoria con respecto a los grados de PTFPI: las pymes que no cuentan con trabajadores FP en estructuras donde desempeñan funciones de desarrollo técnico/tecnológico son las que menos PTFPI muestran, en general. 
Tabla 5. Grado de PTFPI según implicación en prácticas organizativas avanzadas controlando nivel tecnológico

\begin{tabular}{|c|c|c|c|c|c|c|c|c|c|c|c|c|}
\hline \multirow{4}{*}{ PTFPI } & \multicolumn{12}{|c|}{ Nivel tecnológico Medio-Bajo o Bajo } \\
\hline & \multicolumn{6}{|c|}{$\begin{array}{l}\text { Implicación operarios en equipos de trabajo } \\
\qquad a d h o c^{* *}\end{array}$} & \multicolumn{6}{|c|}{$\begin{array}{l}\text { Implicación operarios en reuniones } \\
\text { planificación** }\end{array}$} \\
\hline & \multicolumn{2}{|c|}{ Menos } & \multicolumn{2}{|c|}{ Más } & \multicolumn{2}{|c|}{ Total } & \multicolumn{2}{|c|}{ Menos } & \multicolumn{2}{|c|}{ Más } & \multicolumn{2}{|c|}{ Total } \\
\hline & $n$ & $\%$ & $n$ & $\%$ & $\mathrm{n}$ & $\%$ & $n$ & $\%$ & $\mathrm{n}$ & $\%$ & $n$ & $\%$ \\
\hline Nada & 15 & 16,3 & 4 & 7,0 & 19 & 12,8 & 21 & 20,2 & 5 & 6,9 & 26 & 14,8 \\
\hline Poca & 26 & 28,3 & 10 & 17,5 & 36 & 24,2 & 26 & 25,0 & 14 & 19,4 & 40 & 22,7 \\
\hline Bastante & 24 & 26,1 & 11 & 19,3 & 35 & 23,5 & 28 & 26,9 & 15 & 20,8 & 43 & 24,4 \\
\hline Mucha & 27 & 29,3 & 32 & 56,1 & 59 & 39,6 & 29 & 27,9 & 38 & 52,8 & 67 & 38,1 \\
\hline Total & 92 & 100,0 & 57 & 100,0 & 149 & 100,0 & 104 & 100,0 & 72 & 100,0 & 176 & 100,0 \\
\hline
\end{tabular}

${ }^{*}$ Chi ${ }^{2}$ sig. $<0,01$

Fuente: Elaboración propia

\section{Prácticas organizativas avanzadas y PTFPI}

Profundizando en esta línea de perfiles que nos sugieren la existencia de formas de especialización internas en las pymes, también es interesante la relación entre la PTFPI y otros indicadores obtenidos con la encuesta sobre formas de organización del trabajo.

De un conjunto de prácticas organizativas avanzadas sobre las que se preguntó a las empresas $^{7}$ resultaron dos particularmente interesantes porque, de algún modo, resumían las demás: "operarios implicados en equipos de trabajo temporales

7 También se preguntó sobre las siguientes cuestiones: "Preparan las máquinas que utilizan"; "Realizan el mantenimiento de sus equipos"; "Analizan los datos resultantes de su trabajo"; "Planifican y organizan autónomamente su trabajo"; "Rotan en las tareas"; "Están capacitados para trabajar en diferentes puestos (polivalencia)"; y, "Tienen a su disposición un sistema de recogida de propuestas de empleados/ Buzones de sugerencias". y ad hoc para resolución de problemas (círculos de calidad, kaizen, equipos de mejora" y "operarios intervienen en reuniones periódicas estructuradas de planificación, organización y evaluación del trabajo y resultados, mejoras, etc.". Así, por ejemplo, la menor 0 mayor implicación en alguna de estas dos prácticas presenta una relación directa con la PTFPI. Esta relación, una vez más, solo se produce entre las pymes que pertenecen a sectores de menor intensidad tecnológica (tabla 5).

Como vemos, cuando se trata de empresas que en general desarrollan actividades de nivel tecnológico bajo o medio/bajo, la introducción de estas prácticas organizativas avanzadas hasta los niveles inferiores de la jerarquía (con participación de operarios) está fuertemente vinculada con los más altos grados de PTFPI. En la tabla 5 queda reflejado que en las empresas donde se producen mayores niveles de implicación de los operarios en actividades organizativas avanzadas, se produce también mucha PTFPI (más del $50 \%$ de los casos). 
Otra de las relaciones detectadas como resultado de los análisis efectuados es la que se suscita entre los grados de PTFPI y la intensidad en las actividades de innovación por parte de las pymes. Para ello, tal y como vimos en el primer apartado de resultados dedicado a exponer las características de las empresas que componen la muestra, contamos con un indicador que informa sobre la percepción comparativa en capacidad innovadora. Esta información la hemos complementado con valoraciones de la experiencia en proyectos de cooperación en innovación con agentes externos.

La relación entre PTFPI y capacidad de innovación, tal y como se aprecia en la tabla 6 , está condicionada a la neutralización del nivel tecnológico. Por tanto, es entre las pymes en sectores de nivel tecnológico medio-bajo y bajo donde se aprecia claramente que, en las empresas más innovadoras, destaca la PTFPI en actividades de innovación de producto y proceso. En más de la mitad de las pymes innovadoras (52,5\%) la participación se encuentra a nivel máximo. En los nive- les tecnológicos más altos, la percepción subjetiva de capacidad innovadora tiene una distribución diferente, probablemente, porque la competencia tiene otras exigencias, de manera que son pocas las pymes que se ven en ventaja respecto al resto y, en general, la participación de los perfiles FP es similar.

En cuanto a la cooperación para innovar, en trabajos anteriores (Otero et al., 2014) se ha detectado empíricamente que, tanto en el sistema regional de innovación vasco como en otros (p.e., Cataluña y Navarra), la cooperación representa estadios avanzados de innovación. En esta ocasión, trabajamos sobre una muestra de pymes en la que se detecta un alto nivel de actividades de innovación, que se completa, como era de prever según el argumento anterior, con un importante nivel de cooperación con agentes externos: el $64 \%$ de las empresas consultadas ha tenido esa experiencia de interacción, sobre todo, con proveedores y clientes $(76 \%)$ y también con centros tecnológicos $(66,5 \%)$.

Tabla 6. Grado de PTFPI según percepción de la capacidad innovadora, controlando nivel tecnológico

\begin{tabular}{|c|c|c|c|c|c|c|c|c|c|c|c|c|}
\hline \multirow{4}{*}{ PTFPI } & \multicolumn{6}{|c|}{ Nivel tecnológico Alto o Medio-Alto } & \multicolumn{6}{|c|}{ Nivel tecnológico Medio-Bajo o Bajo } \\
\hline & \multicolumn{6}{|c|}{ Capacidad Innovadora } & \multicolumn{6}{|c|}{ Capacidad Innovadora** } \\
\hline & \multicolumn{2}{|c|}{ Innovadora+ } & \multicolumn{2}{|c|}{ No innovadora } & \multicolumn{2}{|c|}{ Total } & \multicolumn{2}{|c|}{ Innovadora+ } & \multicolumn{2}{|c|}{ No innovadora } & \multicolumn{2}{|c|}{ Total } \\
\hline & $\mathrm{n}$ & $\%$ & $n$ & $\%$ & $n$ & $\%$ & $n$ & $\%$ & $\mathrm{n}$ & $\%$ & $n$ & $\%$ \\
\hline Nada & 2 & 7,7 & 11 & 15,5 & 13 & 13,4 & 9 & 15,3 & 24 & 17,9 & 33 & 17,1 \\
\hline Poca & 10 & 38,5 & 24 & 33,8 & 34 & 35,1 & 11 & 18,6 & 32 & 23,9 & 43 & 22,3 \\
\hline Bastante & 6 & 23,1 & 15 & 21,1 & 21 & 21,6 & 8 & 13,6 & 38 & 28,4 & 46 & 23,8 \\
\hline Mucha & 8 & 30,8 & 21 & 29,6 & 29 & 29,9 & 31 & 52,5 & 40 & 29,9 & 71 & 36,8 \\
\hline Total & 26 & 100,0 & 71 & 100,0 & 97 & 100,0 & 59 & 100,0 & 134 & 100,0 & 193 & 100,0 \\
\hline
\end{tabular}

${ }^{*}$ Chi ${ }^{2}$ sig. $<0,01$

Fuente: Elaboración propia 
Fuente: Elaboración propia

Tabla 7. Grado de PTFPI según Cooperación

\begin{tabular}{lrrrrrrr}
\hline & \multicolumn{9}{c}{ Coopera en innovación** } \\
\cline { 2 - 8 } & \multicolumn{2}{c}{ Sí coopera } & \multicolumn{2}{c}{ No Coopera } & \multicolumn{2}{c}{ Total } \\
\hline Nada & 19 & $9,9 \%$ & 28 & $26,4 \%$ & 47 & 15,8 \\
\hline Poca & 52 & $27,2 \%$ & 27 & $25,5 \%$ & 79 & 26,6 \\
\hline Bastante & 38 & $19,9 \%$ & 31 & $29,2 \%$ & 69 & 23,2 \\
\hline Mucha & 82 & $42,9 \%$ & 20 & $18,9 \%$ & 102 & 34,3 \\
\hline Total & 191 & $100,0 \%$ & 106 & $100,0 \%$ & 297 & 100,0 \\
\hline
\end{tabular}

${ }^{*} \mathrm{Chi}^{2}$ sig. $<0,01$

Fuente: Elaboración propia

Este resultado pone de manifiesto, bien a las claras, la relevancia de la PTFPI. Si, en general, el nivel máximo de participación alcanza a un 34\% de pymes, entre las que han tenido experiencias de cooperación, la PTFPI (producto y proceso) llega a un $43 \%$ de las pymes, bajando hasta un $19 \%$ entre las que no cooperan. Se trataría de una relación significativa entre esta forma de cooperación interna (medida de manera indirecta a partir de la participación de trabajadores con cualificación de FP en actividades de innovación) y la cooperación externa (la colaboración con agentes externos, agentes de I+D u otras empresas) en proyectos de innovación.

\section{Predictores de participación}

En este apartado presentamos los resultados de una regresión logística binaria que nos permite identificar factores diferencialmente asociados al hecho de que las pymes tengan más participación de trabajadores FP en innovación, controlando a la vez los efectos múltiples de un conjunto de variables potencialmente explicativas de la participación ${ }^{8}$. Se han considerado únicamente las variables que inciden efectivamente en los grados de participación (tabla 8).

8 La técnica se utiliza con fines descriptivos, no predictivos. Se han realizado diversos ejercicios de modelación, tanto directos como secuenciales. Aunque los resultados de bondad de ajuste de los modelos no son del todo concluyentes, las asociaciones confirman los análisis previos.
El nivel tecnológico de las pymes ha resultado ser una variable de segmentación necesaria para observar los efectos. Entre las pymes de mayor nivel tecnológico prácticamente no se pueden identificar perfiles multivariantes de estimación de participación: solo la presencia de trabajadores FP en empleos dedicados al desarrollo técnico/ tecnológico se relaciona significativamente de modo positivo con el hecho de que haya mayor participación en innovación. Esta presencia, como mínimo triplica (Exp. $B=3,5$ ) la probabilidad de que una empresa innovadora presente más participación de estos trabajadores a iguales condiciones de tamaño, capacidad 0 intensidad de innovación (coopera).

Es entre las pymes de menor nivel tecnológico donde aparece una combinación de dos tipos de variables como predictoras significativas de la PTFPI; a saber: especialización de la plantilla (FP en empleos técnicos/tecnológicos) y, sobre todo, el nivel superior de pyme innovadora representado por aquellas que desarrollan actividades de cooperación para la innovación. Así las cosas, cooperar triplica la probabilidad de mayor participación y, contar con FP en puestos técnicos, casi la duplica. Frente a estas asociaciones, ni el tamaño, ni la percepción subjetiva de la capacidad innovadora se relacionan con una mayor probabilidad de PTFPI. 
Tabla 8. Modelos de Regresión logística binaria. Predictores de PTFPI (Menor/Mayor) según Nivel tecnológico de las pymes

\begin{tabular}{lcccccc}
\hline & \multicolumn{3}{c}{$\begin{array}{c}\text { Nivel tecnológico Alto } \\
\text { alto }\end{array}$} & medio- & \multicolumn{3}{c}{ Nivel tecnológico Bajo o medio- } \\
bajo
\end{tabular}

Fuente: Elaboración propia

\section{CONCLUSIONES}

La teoría sobre sistemas regionales de innovación pone de manifiesto la importancia de los factores contextuales y geográficamente localizados en los procesos de innovación, especialmente en el caso de las pyme. Entre estos factores cobra un papel importante el sistema educativo y, dentro del mismo, el sistema de FP. En el País Vasco, región característicamente industrial dentro del ámbito español, la FP ha jugado históricamente un papel relevante en la formación del capital humano que se incorpora a la industria regional, caracterizada singularmente por las actividades metalmecánicas. Tal y como hemos podido apreciar existe una mayor orientación del subsistema de FP hacia las necesidades industriales que en otras regiones españolas: mayores tasas de población activa con estudios de FP, fuerte penetración de los estudios industriales y de la FP superior, Administración proclive a la potenciación de la FP, centros activos en formación continua y orientados a la satisfacción de las necesidades de las empresas por trabajar con marcos regulatorios privados. Dado este contexto facilitador de la provisión de capital humano para la industria, la proposición general que se ha pretendido contrastar es si los trabajadores con cualificaciones intermedias (FP) juegan un papel importante en las actividades de innovación empresarial en las pymes vascas.

Los resultados obtenidos ponen de manifiesto la existencia de una importante penetración de personas trabajadoras con perfiles de FP en las 
empresas analizadas, en las que se desarrollan un buen número de actividades de innovación. Asimismo, queda claro que existe un importante nivel de participación de estos profesionales en las actividades de innovación. Este hecho es comprensible dado que, teniendo muchas de las actividades de innovación el modelo DUI como base, la implicación de los trabajadores con cualificaciones intermedias en las operaciones (montaje, mantenimiento, instalación, compras, servicios de asistencia técnica, etc.) en las que se efectúan actividades de mejora e innovación es insoslayable.

Adicionalmente, los resultados obtenidos sugieren que la PTFPI está asociada a la existencia de mayores niveles de formación de los trabajadores (aquellos trabajadores con estudios de FP superiores). Este hecho apoyaría la línea de resultados propuesta por Lundvall et al. (2008), reforzando la idea de la importancia de contar con trabajadores con cualificaciones de FP para la innovación. Los hallazgos efectuados ponen de manifiesto también la importancia de la presencia de estos perfiles formativos en ocupaciones cuya finalidad es el desarrollo técnico/tecnológico (oficinas técnicas, departamentos de I+D y actividades de ingeniería) y más directamente relacionadas con las innovaciones de producto y proceso. Estos resultados sugieren la posibilidad de que las empresas que cuentan con mayor número de personas que poseen estos perfiles en puestos dedicados al desarrollo técnico/ tecnológico podrían verse beneficiadas de la mejora en su "capacidad de absorción".

Por otro lado, los resultados obtenidos sugieren que estos recursos humanos son vitales para la competitividad de las pymes, base de la economía regional. Vemos que es en empresas industriales de sectores metalmecánicos, con menor componente tecnológico y de pequeño tamaño — típicas de la geografía empresarial vasca-, donde se produce una mayor aportación a los procesos de innovación empresariales. Por tanto, cabría pensar, en el mismo sentido que plantea en su informe para la OCDE Stuart Rosenfeld (1998), que en este grupo numeroso de empresas la innovación depende especialmente de profesionales con perfiles de FP, frente a otros sectores que tienen una mayor presencia relativa de ingenieros o titulados superiores.
Tal y como se ha analizado en el marco teórico, distintos estudios han puesto de manifiesto la importancia de la participación de los trabajadores en estructuras que favorecen compartir información, la transferencia de conocimiento y la adopción de decisiones para que se desarrollen procesos de innovación. En este sentido, el trabajo efectuado pone de manifiesto que, cuando las pymes industriales ponen en marcha grupos de trabajo ad hoc (muchas veces relacionados con la resolución de problemas/proyectos concretos) y cuando se implica a los trabajadores en reuniones de planificación, la participación de las personas con perfiles de FP en actividades de innovación es mayor. Por tanto, cabría suponer que las empresas que cuentan con empleados cualificados (FP) podrían mejorar su desempeño innovador introduciendo este tipo de fórmulas de organización participativa.

Una de las principales contribuciones de este trabajo es que la participación de trabajadores con perfiles de FP es mayor en empresas con claro carácter innovador. Entre éstas, hemos encontrado las que tienen un auto-concepto innovador elevado (consideran ser superiores en innovación a la competencia) aún trabajando en actividades con nivel tecnológico medio-bajo y bajo, y aquellas en las que se produce cooperación para la innovación. A nuestro juicio este fenómeno podría estar explicado por la existencia de una cultura de innovación fuerte en las empresas. Rodríguez et al. (2011: 27) definen cultura social de innovación como "el conjunto de valores, normas, costumbres, creencias, ideologías, estilos de vida o códigos de conducta compartidos (...), que permiten sustentar y legitimar actuaciones innovadoras en individuos, grupos y organizaciones". Si bien muchas de las pymes industriales no trabajan expresamente la cultura de la innovación (Olazaran et al., 2009), hay estudios que sugieren que las empresas más innovadoras son aquellas que otorgan mayor importancia a los valores propios de la innovación (García, 2009). Este hecho podría explicar la importancia atribuida por los propios miembros de la empresa a la innovación y a los resultados de la misma, así como a que se desarrollen con naturalidad actividades de cooperación con otros agentes para la innovación. Así las cosas, la participación de los trabajadores 
con perfiles de FP en actividades de innovación podría estar relacionada con la existencia de una cultura de innovación sólida en las empresas, si bien, este es un extremo que habría que confirmar en estudios posteriores.

En el contexto de los trabajos recientes que alertan sobre la importancia de los sectores de menor intensidad tecnológica, frecuentemente relegados en los estudios y políticas de innovación, para la competitividad y el empleo en Europa (Som y Kirner, 2015), la investigación realizada muestra una característica hasta ahora no detectada de dichos sectores: la participación de los trabajadores de FP en los procesos de innovación. Esta participación es un componente importante del modo de innovación de las empresas clasificadas estadísticamente dentro de dichos sectores, y aparece asociada al carácter innovador de las empresas, tanto en su vertiente de generación de nuevo conocimiento interno (e innovación organizativa), como en su vertiente de capacidad de absorción de conocimiento externo, a través de la cooperación con otras empresas y agentes.

Para concluir señalaremos que este estudio permite contrastar la proposición relativa a la aportación de los trabajadores con cualificaciones de FP a las actividades de innovación empresarial en el País Vasco. Los resultados de este estudio están contextualizados dentro del sistema regional de innovación vasco y proceden de una muestra con las características propias de la industria regional. Probablemente, en un contexto diferente, las variables que explican la participación de los trabajadores con perfiles de FP en actividades de innovación serían distintas. Este hecho abre un interesante campo de investigación tendente a identificar qué variables inciden de manera estructural en la participación de estos profesionales en la innovación y cuáles otras son contextuales.

\section{AGRADECIMIENTOS Y FINANCIACIÓN}

Este trabajo ha sido realizado dentro del proyecto "Formación profesional y sistema de innovación: El papel de los trabajadores intermedios en los procesos de innovación de las pymes industriales", financiado por el
Ministerio de Ciencia e Innovación, Gobierno de España (CS02011-29410-C03-01). Así mismo, en las fases preliminares y actividades complementarias, el equipo contó con la ayuda a grupos de investigación IT593-13 del Departamento de Educación, Universidades e Investigación del Gobierno Vasco.

\section{REFERENCIAS BIBLIOGRÁFICAS}

Albizu, E., Olazaran, M., Lavía, C., Otero, B. (2013). Comunidad Autónoma del País Vasco. En M. Olazaran, I. Brunet, Entorno regional y formación profesional: los casos de Aragón, Asturias, Cataluña, Madrid, Navarra y País Vasco (pp. 189-217). Tarragona: Publicacions URV y Servicio Editorial UPV/EHU.

Béduwè, C., Planas, J. (2003). Educational Expansion and Labour Market:EDEX. Luxemburgo: Office for Official Publications of the European Communities.

Bolland, E. J., Hofer, C. W. (2001). Las empresas del futuro: Cómo funcionan las compañías de alta tecnología en los Estados Unidos de América. México: Oxford University Press.

Chesbrough, H. (2003). Open innovation: The new imperative for creating and profiting from technology. Boston (MA): Harvard Business School Press.

Cohen, W. M., Levinthal, D. A. (1990). Absorptive capacity: a new perspective on learning and innovation. Administrative Science Quarterly, 35, 128-152.

Drucker, P. F. (2000). La productividad del trabajador del conocimiento: máximo desafío. Harvard Deusto Business Review, 98, sept./oct., 4-16.

Eustat (2015). Panorámica de la industria vasca 2015 (en línea). http://www.eustat.eus/elementos/ele0012500/ti_panorama-de-la-industria-vasca-2015/inf0012565_c.pdf, acceso 6 de julio de 2015.

García Espejo, I. (2009). Las relaciones entre innovación, nuevas formas de organización del trabajo y políticas de recursos humanos: el caso de la industria asturiana. Empiria, Revista de metodología de ciencias sociales, 17 (enerojunio), 63-90. 
García-Pinto, A., García, J. M., Piñeiro, P. (2010). "Incidencia de las políticas de recursos humanos en la transferencia de conocimiento y su efecto sobre la innovación". Investigaciones Económicas en Dirección y Economía de la Empresa, 16(1), 149-163.

Gobierno Vasco (2014). IV Plan Vasco de Formación Profesional: hacia una FP diferente. VitoriaGasteiz: Departamento de Educación, Política lingüística y Cultura.

Janz, B. D., Prasarnphanich, P. (2003). Understanding the antecedents of effective knowledge management: the importance of knowledge-centered culture. Decision Sciences, 34(2), 351-384.

Jiménez-Jiménez, D., Sanz-Valle, R. (2008). Could HRM support organizational innovation? The International Journal of Human Resource Management, 19(7), 1208-1221.

Kaufmann, A., Tödtling, F. (2000). System of innovation in traditional industrial regions: The case of Styria in comparative perspective. Regional Studies, 34(1), 29-40.

Lorenz, E., Valeyre, A. (2006). Organisational innovation, human resource management and labour market structure: a comparison of the EU15. The Journal of Industrial Relations, 47(4), diciembre, 424-442.

Lundvall, B. Ã. , Lorenz, E. (2010). Innovación y desarrollo de competencias en la economía del aprendizaje. En D. Parrilli (Coord.), Innovación y aprendizaje: lecciones para el diseño de políticas (pp. 44-101). Zamudio: Innobasque Agencia Vasca de la Innovación, 44-101.

Lundvall, B. Ã., Rasmussen, P., Lorenz, E. (2008). Education in the Learning Economy: a European perspective. Policy Futures in Education, 6(6), 681-700.

Ministerio de Educación, Cultura y Deporte (2014). Las cifras de la Educación en España (en línea). http://www.mecd.gob.es/dms/mecd/serviciosal-ciudadano-mecd/estadisticas/educacion/ indicadores-publicaciones-sintesis/cifras-educacion-espana/2014/D6p.pdf, acceso 7 de julio de 2015.

Moodie, G. (2006). Vocational education institutions'role in national innovation", Research in Post-compulsory Education, 11(2), 131-140.
Navarro, M. (2009). Los sistemas regionales de innovación. Una revisión crítica. Ekonomiaz, 70(1), 24-59.

Nonaka, I. (1994). A dynamic theory of organizational knowledge creation. Organization Science, 5(1), 14-37.

Olazaran, M., Albizu, E., Otero, B. (2009). Innovación en las pequeñas y medianas empresas industriales guipuzcoanas. Leioa: Ed. Servicio Editorial de la Universidad del País Vasco/Euskal Herriko Unibertsitatea.

Otero, B., Lavía, C., Albizu, E., Olazaran, M. (2014). Políticas públicas y cooperación con agentes externos en procesos de innovación: estudio comparado de pymes industriales en tres sistemas regionales. Revista de Dirección y Administración de Empresas, 21, 1-20.

Pérez, C., Quevedo, P. (2006). Human resources management and its impact on innovation performance in companies". International Journal of Technology Management, 35(1, 2, 3, 4), 11-28.

Planas, J.; Sala, G., Vivas, J. (2004). Nuevas estrategias formativas ante el fin de la expansión educativa en España. Revista de Educación, 333(1), pp. 445-461.

Rodríguez, A., Hoyos, J., Izaguirre, J., Vicente, M. A. (2011). Organizaciones en el marco de una cultura social innovadora: Propuesta de factores explicativos. Investigaciones Europeas de Dirección y Economía de la Empresa, 17(1), enero-abril, 17-35.

Rosenfeld, S. (1998). Technical colleges, technology deployment and regional development. Modena: OECD.

Shavit, Y., Muller, W. (1998). From School to Work: A Comparative Study of Educational Qualifications and Occupational Destinations. Oxford: Clarendon Press.

Som, 0., Kirner, E. (2015). Low-tech Innovation: Competitiveness of the German Manufacturing Sector. Springer International Publishing.

Toner, P., Marceau, J., Hall, R., Considine, G. (2004). Innovation Agents: VET Skills and Innovation in Australian Industries and Firms (Volume 1). Adelaida: National Centre for Vocational Education Research (NCVER). 
Zahra, S. A., George, G. (2002). "Absorptive capacity: a review, reconceptualization and extension". Academy of management review, 27, 185-203.

\section{NOTAS BIOGRÁFICAS}

Beatriz Otero Gutiérrez (Santander, 1978) es doctora en Sociología por la UPV/EHU y profesora adjunta en el departamento de Sociología y Trabajo Social de la misma universidad. Ha participado en diferentes proyectos y publicaciones en el ámbito de los sistemas de I+D, la innovación y la educación, siendo el análisis de datos e indicadores de ciencia y tecnología un área de interés en sus investigaciones.

Cristina Lavía Martínez (Bilbao, 1963) es doctora en Sociología por la Universidad de Deusto y actualmente profesora titular en el Departamento de Sociología y Trabajo Social de la Universidad del País Vasco (UPV/EHU). Sus líneas de investigación y publicación se centran en las metodologías cuantitativas, el desarrollo de nuevos indicadores sociales así como en el análisis de sistemas de innovación.

Mikel Olazaran Rodríguez (Pamplona, 1963) es doctor en Sociología por la Universidad de Edimburgo (1991). Trabaja como profesor titular en el departamento de Sociología y Trabajo Social de la UPV/EHU. Ha realizado investigaciones y publicaciones en torno a la innovación, las organizaciones y la educación. Entre 2005 y 2012 fue decano del Colegio Oficial de Doctores y Licenciados en Ciencias Políticas y Sociología de Navarra.

Eneka Albizu Gallastegi (Bilbao, 1963) es doctor en Economía /UPV/EHU), catedrático en la Facultad de Relaciones Laborales y Trabajo Social y director del Instituto de Economía Aplicada a la Empresa. Ocupa un puesto de catedrático en la Escuela Universitaria de Relaciones Laborales de la misma universidad. Sus áreas de interés investigador pivotan, fundamentalmente, sobre la innovación, tanto en lo concerniente al cambio y aprendizaje organizativo, como a la gestión de personas.

\section{ANEXO: CONTENIDO DEL CUESTIONARIO UTILIZADO}

La encuesta telefónica realizada se planificó para ser realizada en 15 minutos por personal profesional entrenado. El cuestionario utilizado para llevar a cabo la encuesta telefónica se grabó en CATI y constaba de siete bloques:

\section{Bloque 0: datos generales de la empresa.}

En este grupo se confirmaban los datos de la empresa disponibles en la base de sondeo y se añadían otros nuevos. En concreto se preguntaba por el tamaño (número de empleados), sectores de actividad (CNAE09), volumen de actividad exportadora (\% sobre facturación), antigüedad, y tipo de proceso productivo. Asimismo, se pedía una valoración de la situación competitiva de la empresa respecto a sus competidores directos (peor, igual o mejor).

\section{Bloque 1: sobre los trabajadores con perfil FP en la plantilla.}

En este bloque se preguntaba por la cantidad de trabajadores con estudios de FP en la empresa (\% sobre el total) y por la cantidad de ellos que tenían el nivel FP superior (\% sobre el total de trabajadores FP). También se pedía el porcentaje de trabajadores FP entre el personal de distintos niveles jerárquicos agrupados en cuatro: alta dirección y direcciones departamentales; mandos intermedios; empleados administrativos y comerciales 0 similares; y operarios.

\section{Bloque 2: actividades desarrolladas por empleados con perfil FP.}

Especificando una serie de hasta doce actividades productivas y de otro tipo que se pueden desarrollar en pymes industriales, se preguntaba primero si existían y caso de existir, qué porcentaje de personal participante tenía el nivel FP. Las actividades incluían las oficinas técnicas, departamentos de I+D, ingenierías de fabricación y procesos, mantenimiento de maquinarias, prevención y riesgos, operaciones de transformación, montaje, instalación y asistencia técnica (SAT), calidad, recursos humanos, compras y logística. 


\section{Bloque 3: formación en la empresa.}

Las preguntas se dirigían a averiguar cuántos empleados habían recibido formación fuera del puesto de trabajo (\% en los últimos 2 años), qué porcentaje del gasto había sido subvencionado por las administraciones públicas y cuántas horas por término medio (empleado/año) de formación habían recibido.

\section{Bloque 4: actividades auxiliares y prácticas organizativas de los operarios.}

Sobre una lista de nueve actividades de este tipo se preguntaba si existían o no y hasta qué punto participaban los operarios (nada, poco, bastante, mucho): preparar sus máquinas; mantenimiento de sus equipos, analizar datos de su trabajo; planificar y organizar autónomamente su trabajo; rotar en tareas; ser polivalentes; tener un sistema de propuestas; participar en equipos "ad hoc" para resolución de problemas; y participar en reuniones periódicas de planificación y mejora.

\section{Bloque 5: actividades de innovación y mejora y participación de empleados con FP.}

Se pregunta si la empresa innova (según la clasificación estadística) en proceso, producto, orga- nización y mercado 0 si realiza mejoras en productos 0 procesos. Para cada actividad de innovación se averigua cuánto participan los empleados con perfil FP. También se plantea una batería de cinco posibles factores que dificultarían esa participación para que se les asigne grado de importancia. En este mismo bloque se pregunta si la empresa ha cooperado en proyectos de innovación, si es así con qué agentes externos y se le pide una valoración global de su capacidad innovadora frente a la competencia (peor, igual, mejor).

\section{Bloque 6: valoración de la oferta de Formación Profesional en el entorno.}

Se averigua en qué consisten las relaciones con centros de FP del entorno (recibir alumnado en prácticas, contratar con sus bolsas, recibir formación de oferta y/o de demanda, recibir asistencia técnica, ceder maquinaria). Se piden valoraciones de la oferta de FP, la formación que se da, la capacitación resultante y, finalmente, se pregunta directamente si la empresa considera que la contribución de sus recursos humanos con formación FP es un soporte relevante para la posición competitiva de la empresa. 